\section{The GDC - lifting the lid. Part 2: registration}

\author{
H. Mathewson ${ }^{1}$ and D. Rudkin²
}

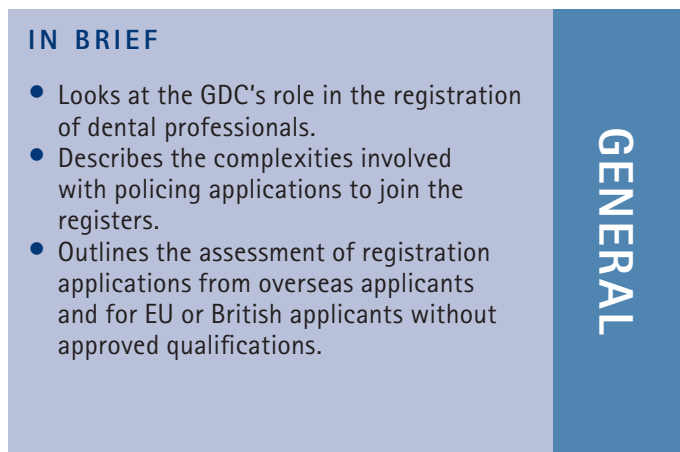

This second part of the BDJ series on the work and future plans of the General Dental Council looks at the GDC's role in the registration of dental professionals. To dentists, registration is perhaps the most immediately obvious aspect of the GDC's work. The article gives an in-depth look at this important area, introducing the reader to some of those involved with registration enquiries and looking at some of the more complex issues involved with registration, including the process of dealing with registration applications from overseas.

In our first article we gave you a glimpse into the day to day workings of the GDC's standards team. We can't go any further without shining a light on the GDC register. A great, dusty, leather-bound tome guarded by a whiskery clerk straight out of a Dickens novel? Actually, and shockingly, we were still using leather-bound tomes until quite recently. Although the medium of record has changed from quill pen and parchment to database and mouse, the register continues to represent a set of promises we make to the public about the people on it. Picturesque Dickensian clerks have long since given way to a new generation of expert, motivated and professional GDC staff.

THE GDC - LIFTING THE LID
1. Professionalism and standards
2. Registration
3. Education, CPD and revalidation
4. Fitness to practise
5. Illegal practice

President, ${ }^{2 *}$ Chief Executive and Registrar, General Dental Council, 37 Wimpole Street, London, W1G 8D0 ${ }^{*}$ Correspondence to: Mr Duncan Rudkin Email:drudkin@gdc-uk.org

Refereed Paper

DOI: 10.1038/sj.bdj.2008.465

${ }^{\oplus}$ British Dental Journal 2008; 204: 639-641
Take Sangeeta Dhanji (Fig. 1), a member of a team of seven dealing with customer enquiries and for many, the first voice callers hear when they contact the GDC. 'I can get more than 70 enquiries a day on the phone, and nearly as many emails,' says Sangeeta, who came to us from the General Medical Council. She and her colleagues, who also welcome visitors at reception, log around 7,000 calls and emails a month. Many are about the register.

'Members of the public, registrants, other organisations - I deal with them all,' says Sangeeta. 'I give advice and guidance on the role and function of the Council, its procedures and processes, so I have to keep up to date on the Council's guidance and policies. The register is a key aspect of this.'

Often, dental professionals want to know how to register - they may be a UK graduate, or have qualified in Europe or further afield; they might want information about getting back on the register, perhaps after travelling overseas; or they may even want to know how to get off the register, maybe for a career break.

\section{Registration for those without approved qualifications}

Anyone who qualified in the UK has an automatic right to apply for registration. The only approved qualifications are

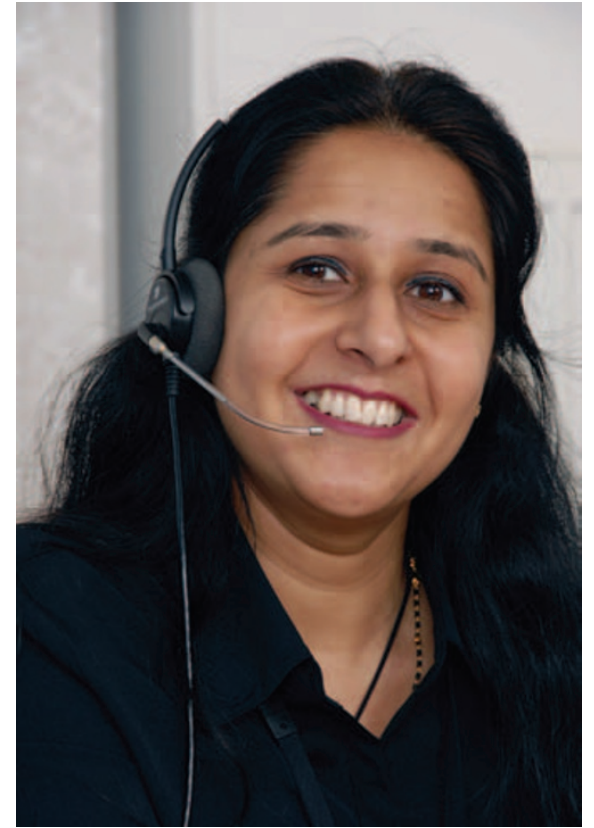

Fig. 1 Sangeeta Dhanji, a member of the GDC customer enquiries team

those from the UK and various scheduled EU qualifications. So what about dental professionals who don't have one of these qualifications?

'We tease out whether applicants are eligible to register - whether they "demonstrate the knowledge and skills required", in the words of the 1984 Dentists Act - and if not, how they might move forward,' says Phil Higgs, who has, for two years, managed a small team assessing the more challenging applications.

These applications require a good deal 
more consideration and assessment than standard applications. The applicant may be a European national who has qualified outside Europe, with or without a postgraduate qualification, and if the former, with varying degrees of postgraduate experience. The touchstone is whether they can show they meet UK standards.

'A small minority do embellish their experience. Of course, 90\% of applications are straightforward, and it's those applicants - and existing registrants - who gain when we scrutinise the $10 \%$ of applicants who may have sometimes minor flaws in their application, or who don't achieve the benchmark standards.'

Phil's 15 minutes of GDC fame came when he exposed a fraudulent application from a British national who claimed to have qualified in Scandinavia. 'It rang alarm bells, so we made enquiries and found that he had claimed over there that he had registered here.' The police are investigating. 'I feel proud of my role in it all. As a fraudulent registrant, he could clearly have posed a danger to the public.'

From beyond Europe's borders come further applications, to sit the Overseas Registration Examination or ORE.

Whether from Europe or beyond, all applications are assessed individually, rigorously and sensitively. We certainly don't want to obstruct people from registering, but we do need to make sure they have the necessary qualifications and experience to practise in the UK. Decisions can be complex, time consuming and difficult, finely balanced between the rights of an individual and the need to ensure we protect patients.

\section{Transforming the delivery of registration}

Registration is your licence to practise, and the public's guarantee that you are a professional, with the skills and experience to match. We are relentlessly focussing a lot of our time and energy on transforming the way in which we deliver this core service to the public.

When Edward Bannatyne (Fig. 2) came to the GDC as its new director of operations in the summer of 2007, part of his brief was to beef up the process of registering dental nurses and dental technicians. We made heavy weather

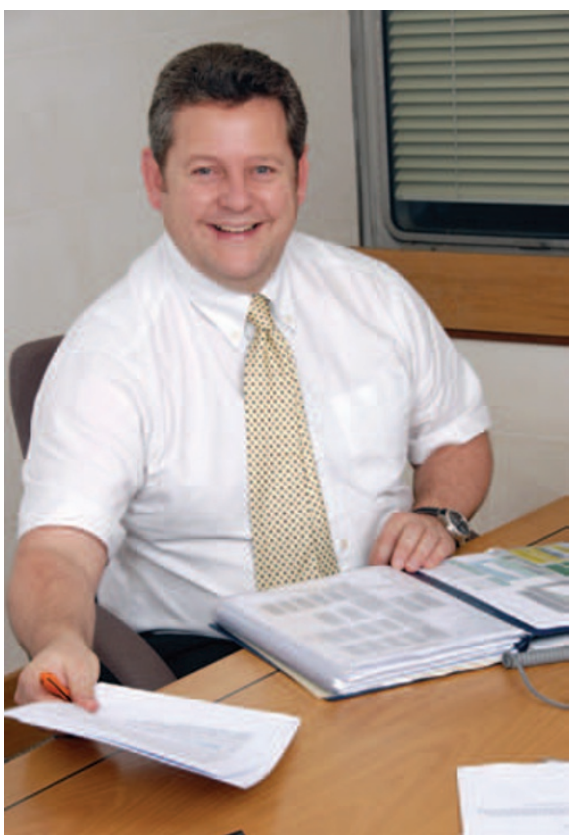

Fig. 2 Edward Bannatyne, GDC Director of Operations

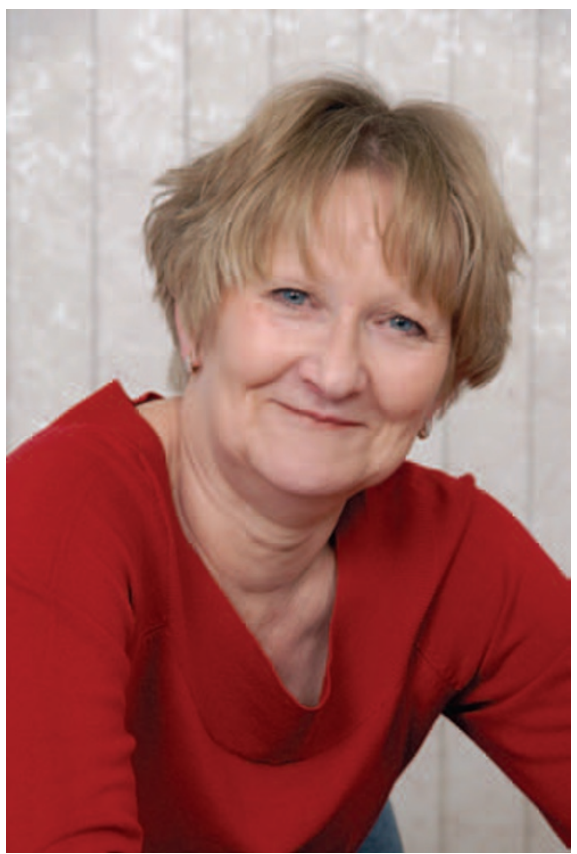

Fig. 3 Ann Kidwell leads the GDC customer enquiries team

of this at first. Application forms were long-winded and off-putting. Processing them was taking much too long.

Building in crucial quality assurance, Edward began streamlining processes. His team worked evenings and weekends to slash backlogs. Huge sackloads of applications were logged. 'We are getting there,' Edward puts it modestly. It is as though a whirlwind dropped by, with prodigious results.

Now, if you request an application form today, it's despatched tomorrow.
We have in the past collected application fees the same day, although the success of DCP registration - the number of forms coming in - has temporarily lengthened response times. Once someone is registered, their certificate goes out within a day, or at the most, two.

You may say, so what? Well, it demonstrates that we are committed to efficiency. We can also use the lessons we have learned in registering DCPs quickly and efficiently to improve the process of registering dentists and others. It is win-win all round. Most importantly of all, the public get more accurate and up to date information about dentists and dental care professionals, which is one of the key reasons for having a register in the first place.

\section{Customer enquiries}

A similar 'virtual circle' of quality assurance happens when it comes to customer enquiries. The customer advice and information team keeps the GDC up to date on issues and concerns raised by GDC customers. Are customers asking for information that hasn't been made easily available? Are the GDC ensuring that they are open and accountable?

The majority of enquiries last year came from dental nurses and dental technician applicants, explains Ann Kidwell (Fig. 3), who leads the enquiry team Sangeeta works in. 'Callers' questions range from why they need to register, to whether they'll get more salary if they do.'

One of the things Edward Bannatyne is doing is trying to improve the "right first time' rate, and Ann is on the front line. Every application with information missing means delay and frustration for applicants. Ann, Sangeeta and their colleagues can talk customers through the forms step by step if that's what they want, fully explaining the requirements and helping to make sure that the applications are completed correctly. Original documents should not be sent in, for instance - certified copies of certificates are what we ask for.

The detail can get complicated but the rules are clear and specific. More challenging sometimes are those calls where the person is having a debate - genuine or otherwise - about whether they need 
to apply for registration at all. This is particularly an issue with some dental technicians. 'People sometimes ask me what are the benefits of getting registered,' says Ann. 'I have to tell them that it's for the public's benefit. Some dental technicians seem to think we're a club for dentists. Not many dentists make that mistake. Funny that.'

\section{Some more members of our team}

Patrick Kavanagh (Fig. 4) manages our registration policy team, which includes keeping on top of the latest European directives and how they affect registration. 'We will be looking again at health checks - whether we should be doing these at all, or whether they should be more stringent.' As always, protecting patients is key.

'We may also need to clarify how to get back on the register if you've been off it for a while, and temporary registration for those who qualified outside the UK and EU: what do we register them to do? And with temporary and occasional workers, it's still unclear what the EU allows us to check.' Paper research is important, of course, but so is monitoring other regulators' best practice.

Krutika Patel is one of the Assessments Team, responsible for processing registration applications. 'I get 20-25 a day. Two thirds of applicants get the documentation right first time. But language and culture can be issues. For instance, we ask for certified copies of documents, but in Germany, Switzerland and Italy, any professional can certify documents, not just a lawyer.'

Potential registrants sometimes try to certify themselves. "They say, "but I am of good character. I am healthy." In the end, it's not bureaucracy, it's protecting the public. We're the regulatory body and have to maintain standards. We just need a standard four core documents, or five with a change of name.'

Andrew Futter (Fig. 5) is currently managing the team that assists applicants for the Overseas Registration Examination (ORE). 'The first step is to ensure that the ORE is the most appropriate route for the applicant - it's open to qualified overseas

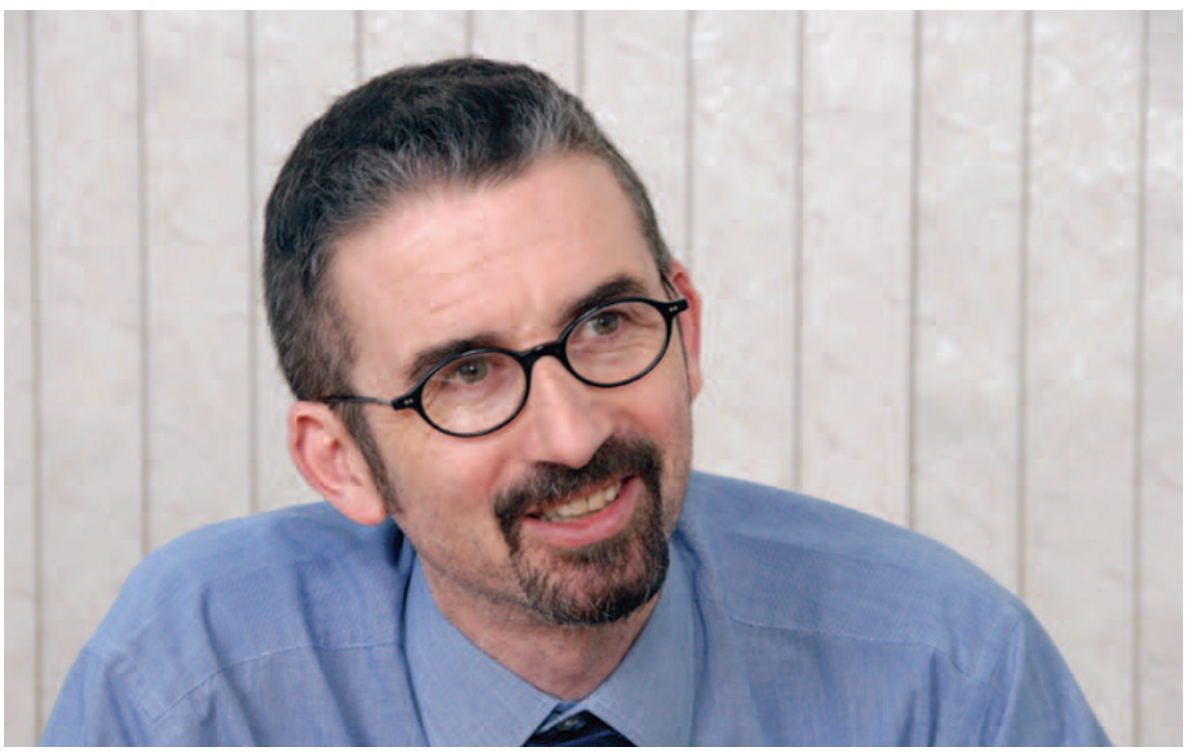

Fig. 4 Patrick Kavanagh, manager of the registration policy team

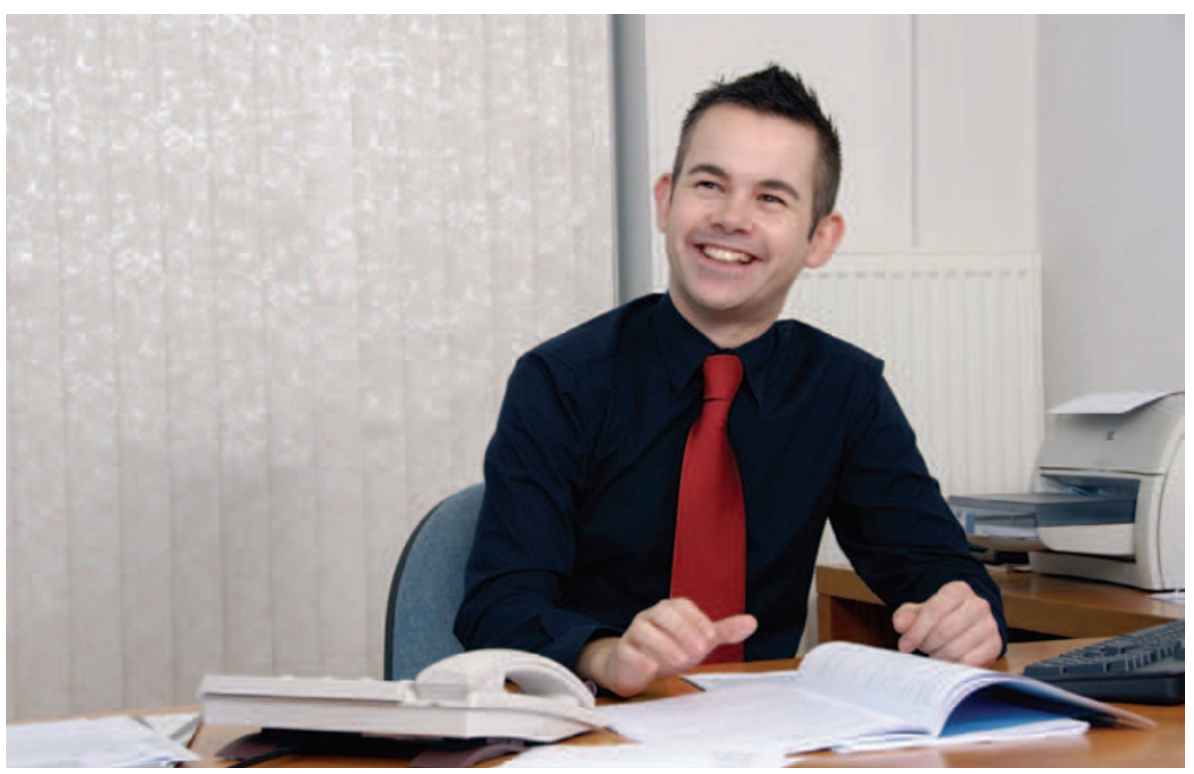

Fig. 5 Andrew Futter manages the team assisting applicants for the Overseas Registration Examination

dentists with 1,600 hours or more practical clinical experience. We require certified copies of an applicant's qualification and their passport, along with the results of an English language test and a certificate of good standing from their current dental regulator.'

Andrew and his colleagues have to be alert and ensure attention to detail. 'If anything looks unusual, we always verify the source of the document and speak to whoever has put their signature to it on behalf of the candidate. To do anything less would be to place the health and safety of patients at risk, and undermine the integrity and purpose of the examination.' One applicant paid $£ 200$ for a forged language qualification. Another forged one from scratch. Neither apparently realised that the GDC examinations team can, and does, check qualifications.

With our complex rules and forms, we do know we can look like a bureaucracy - a more or less efficient one, depending on your point of view. But all this activity is never anything but a means to an end. We aim to be confident when we make promises about you to the public - so that they can have confidence in you. 